\title{
Germination et croissance de deux renoncules aquatiques
}

\author{
Odile Decamps ${ }^{1}$
}

Mots clés: Ranunculus, germination, croissance, rivière.

La faculté germinative de Ranunculus penicillatus var. penicillatus atteint $80 \%$ au bout de 2 à 3 mois avec un maximum plus élevé dans l'eau de la Truyère que dans celle du Lot dont la conductivité est plus élevée. Une germination plus lente caractérise Ranunculus flutans, avec un maximum de $45 \%$ at teint dans l'eau du Lot au bout de 7 mois. La longévité du pouvoir germinatif de Ranunculus penicillatus var, penicillatus dépasse deux ans et demi. Par leur abondance, les racines adventives constituent, sur substrat hétérogène des souches rhizornateuses dès le début de la croissance.

Germination and growth of two aquatic Rantunculus species

Keywords: Ranunculus, germination, growth, river.

The germinative power of Ranunculus penicillatus var. penicillatus is $80 \%$ after 2 to 3 months with a higher maximum in water from the Truyère river than in water from the Lot river which has a higher conductivity. A slower germination characterizes $R$. fluirans, with a maximum of $45 \%$ in water from the Lot river after 7 months. The longevity of the germinative power of $R$. penicillatus var. penicillatus is greater than two and half years. Due to their abundance, the adventive roots form root stocks on a heterogenous substratum at the beginning of growth.

\section{1. - Introduction}

Deux espèces de renoncules aquatiques dominent largement dans les herbiers à macrophytes qui encombrent les cours du Lot et de la Truyère aux environs d'Ent raygues (Décamps \& al. 1979). Il s'agit de Ranunculus penicillatus var. penicillatus (Dumort.) Bad. et Ranunculus fluitans Lam. Depuis longtemps, les auteurs ont souligné l'aptitude de ces espèces à la multiplication végétative. Cette aptitude est d'ailleurs considérée d'une manière classique, comme responsable de la prolifération des espèces aquatiques de la famille des Renonculacées (Whitton \& Buckmaster 1970, Westlake 1973). Cependant, ces renoncules germent (Arber 1920, Cook 1966) et on ne doit pas sous-estimer les possibilités d'expansion à partir des germinations au moins dans certains cas. Mais ce domaine de la biologie des renoncules aquatiques demeure encore mal connu. C'est pourquoi les deux espèces de Ranunculus récoltées en abondance dans les cours du Lot et de la Truyère a ux

1. Laboratoire botanique et forestier, Université Paul Sabatier 39, allées Jules-Guesde, 31062 Toulouse. environs d'Entraygues ont fait l'objet d'une série d'expériences dans le but de préciser les conditions de germination, de longévité de cette germination et de croissance des plantules dans le milieu naturel et au laboratoire.

\section{2. - Stations étudiées et méthodes}

Les deux stations étudiées sont situées sur la Truyère et sur le Lot à Entraygues en amont de leur confluent (Laville 1981). L'ensemble des analyses montre que ces deux rivières different nettement dans leurs caractéristiques chimiques. Rappelons simplement les valeurs suivantes (moyennes arithmétiques des mesures prises de 1971 à 1975 et L.C. à P. 0,05) d'après Capblancq et Tourenq (1978) :

$$
\text { Truyère Lot }
$$

Conductivité

$$
\begin{aligned}
& \text { ( } \mu \text { mhos. } \\
& \left.\mathrm{cm}^{-1} 20^{\circ}\right) \quad 56,25 \pm 6,12 \quad 167,5 \pm 13,1 \\
& \mathrm{HOO3}^{-} \text {(mél -l) } 0,464 \pm 0,07 \quad 1,588 \pm 0,08 \\
& \left.\mathrm{CA}^{++} \text {(mél }-1\right) \quad 0,270 \pm 0,055 \quad 1,220 \pm 0,234 \\
& \mathrm{Si} \mathrm{O}_{2}(\mathrm{mgl}-1) \quad 8,42 \pm 1,17 \quad 5,40 \pm 1,0
\end{aligned}
$$


La Truyère (débit annuel moyen: $61 \mathrm{~m}^{3} \mathrm{~s}^{-1}$ ) qui draine en effet un bassin cristallin présente des eaux plus acides et trois fois moins minéralisées en moyenne que les eaux du Lot (débit annuel moyen : $34,8 \mathrm{~m}^{3} \mathrm{~s}^{-1}$ ) qui, elles, proviennent d'un bassin versant à dominante calcaire. Les eaux de la Truyère se distinguent encore de celles du Lot par des teneurs en silice plus élevées.

En amont du confluent, les herbiers de la Truyère comprennent en abondance Ranuncultis penicillatus var. penicillatus tandis que le Lot abrite en moindre abondance essentiellement l'espece Ranunculus fluitans en amont et en aval du confluent.

Au laboratoire, les semences ont été mises en aquarium, soit directement prélevées à la station et transportées dans l'eau, soit prélevées et réensemencées après des périodes de déshydratation plus ou moins longues. Ces semences ont été placées sur un substrat de sable des berges, lavé et stérilisé, avec une alimentation régulière en eau de la station ou en eau distillée suivant les expériences.

\section{3. - Résultats et discussions}

\section{1. - Faculté germinative}

Pour chacune des deux espèces, des lots de 100 graines ont été placés en aquarium dans le but d'obtenir des germinations de plantules en différents milieux : eau du Lot, eau de la Truyère, eau distillée. Les plantules ont été comptées régulièrement. Les courbes de germination sont trés différentes lorsqu'on compare les deux espèces (fig. 1).

En effet, la faculté germinative est importante et peut at teindre $80 \%$ des graines immergées pour $R$. penicillatus var. penicillatus. Le début de la germination a lieu 20 à 30 jours après le semis pour les lots non déshydratés, période à laquelle il faut ajouter 7 à 15 jours pour permettre la réhydratation des lots déshydratés. Par ailleurs, toutes les semences d'un même lot ne commencent pas à germer en même temps: le maximum de germinations est atteint au bout de deux ou trois mois. Comme le montre la figure 1, ce maximum est plus élevé dans l'eau de la Truyère (où cette espece prolifère) que dans l'eau du Lot ; il est beaucoup plus faible en eau distillée.

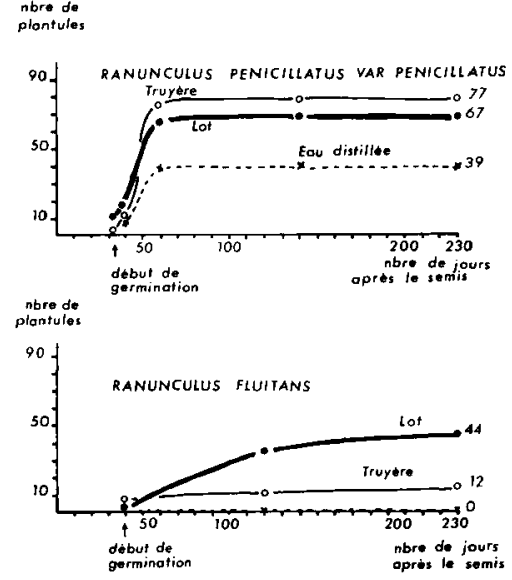

Fig. 1. Courbes de germinations obtenues par des lots de 100 graines de Ranunculus penicillatus var. penicillatus et de Ranunculus fluitans mises à germer respectivement dans de l'eau du Lot, de la Truyère et dans de l'eau distillée.

Une germination beaucoup plus lente caractérise Ranunculus fluitans dont certaines graines déshydratées peuvent ne germer que sept mois après immersion. Le maximum de germination est bien inférieur à celui de l'espèce précédente puisqu'il atteint 45 sur 100 graines immergées dans l'eau du Lot et 13 sur 100 graines immergées dans l'eau de la Truyère. Ranunculus fluitans paraît être une espèce sensible aux conditions de milieu. Whitton \& Buckmaster (1970) avaient déjà souligné sa sensibilité aux eaux polluées par l'industrie du coke. Contrairement aux résultats obtenus pour $R$. penicillatus var. penicillatus c'est dans l'eau du Lot, et non de la Truyère (c'est-à-dire dans de l'eau provenant de son milieu d'origine) que $R$. flutians atteint son pourcentage de germination le plus élevé. Il faut souligner aussi que cette espèce fleurit plus difficilement. Remarquons que des graines de $R$. fluitans, variété à feuilles étroites peuplant les eaux de la Dordogne ont un pourcentage de germination également plus élevé dans les eaux du Lot que dans celles de la Truyère. La plantule a par ailleurs le même 
développement hétéroblastique que celle de $R$. fluitans, le caractère feuilles étroites n'apparaissant que chez l'adulte des eaux de la Truyère.

L'observation des courbes de la figure 1 fait encore apparaître un phénomène portant sur un nombre de graines peu important, mais remarqué de façon constante dans toutes les expériences couplées : au début de la germination, le plus fort taux de germination est observé pour des milieux distincts des milieux d'origine (eau du Lot pour Ranunculus penicillatus var. penicillatus, eau de la Truyère pour Ranunculus fluitans) la tendance s'inverse ensuite rapidement bien avant que le maximum de germination soit atteint.

\section{2. - Longévité du pouvoir germinatif}

Les expériences ont été conduites sur l'espèce $R$. penicillatus var. penicillatus. Plusieurs lots de 100 graines prélevées sur place en août-septembre de 1977 à 1979 dans la Truyère ont été conservés à sec afin de tester la longévité du pouvoir germinatif. Ces graines ont ensuite été remises en eau immédiatement ou après des périodes d'assèchement de 1,2 , $3,6,12,18,24$ et 30 mois (tableau 1). Ces expériences montrent que des pourcentages de germinations assez proches, $71 \%$ en moyenne, caractérisent les semences stockées pendant les 3 premiers mois. Ces pourcentages s'abaissent pour des semences stockées pendant 12 mois à 24 mois de 53 à $42 \%$ en moyenne et pour des semences stockées pendant 30 mois à $26 \%$. Des graines échouées sur les berges après une crue ou transportées par des oiseaux ou autres agents de dissémination peuvent donc germer à la faveur de remises en eaux après un, deux ou deux ans et demi ; en fait, l'omithochorie peut être responsable de la colonisation en amont des stations existantes ou des réservoirs latéraux. Ceci peut expliquer certaines expansions telles que celles observées sur le cours d'une rivière comme le Lot pendant ces dernières années.

\section{3. - Modalités de la croissance et cycle}

Un autre caractère important est la capacité de ces plantes à s'adapter, notamment grâce à diverses modalités de croissance, aux conditions du milieu. On peut dire que leur plasticité est extrême ce qui d'ailleurs n'est pas propre au genre Ranunculus (Batrachium D.C., A. Gray) dans la famille des Renonculacées (Décamps 1978, 1979). Un autre exemple frappant est celui de Ranunculus thora L., espèce à faible pouvoir germinatif qui présente des plantules tantôt dicotylées, tantôt monocotylées (Décamps 1972).

En ce qui concerne les renoncules aquatiques, nos observations ont porté sur la croissance des plantules en aquarium d'une part et d'autre part à la station essentiellement sur des touffes de Fontinalis antipyretica qui, substrat d'élection pour les graines de renoucules, permettaient des recensements faciles.

Les expériences faites au laboratoire sont regroupées dans le tableau II qui indique les nombres de feuilles et de racines adventives observées sur des plantules de $R$. penicillatus var. penicillatus âgées de 140 jours et cultivées selon différents mélanges d'eau du Lot et de la Truyère.

Sur le terrain, en complément aux résultats consignés au tableau II, le 20 septembre, sur 27 plantules observées, ont été trouvées : 7 plantules au stade cotylédonaire, 5 à une feuille, 6 à deux feuilles, 4 à trois feuilles et 5 à quatre feuilles. Le

Tableau I. - Longévité du pouvoir germinatif de Ranunculus penicillatus var, penicillatus; nombre de germinations obtenues (dans chaque cas sur 100 graines) après des périodes d'assèchement allant de 0 (nulle) jusqu'à 30 mois.

\begin{tabular}{clllllllll}
\hline $\begin{array}{c}\text { Année de } \\
\text { prélèvement }\end{array}$ & nulle & 1 mois & 2 mois & 3 mois & 6 mois & 12 mois & 18 mois & 24 mois 30 mois \\
\hline 1977 & 58 & 58 & 57 & 56 & 53 & 41 & 38 & 33 & 20 \\
1978 & 74 & 76 & 76 & 74 & 69 & 55 & 48 & 42 & 25 \\
1979 & 82 & 80 & 80 & 80 & 75 & 63 & 55 & 50 & 32 \\
1979 & 72 & 72 & 71 & 70 & 67 & 53 & 47 & 43 & 28 \\
& $71,5 \%$ & $71,5 \%$ & $71 \%$ & $70 \%$ & $66 \%$ & $53 \%$ & $47 \%$ & $42 \%$ & $26 \%$ \\
\hline
\end{tabular}


20 décembre, c'est-à-dire au même âge environ que les plantules observées en aquarium (tableau II), 54 plantules observées se répartissent comme suit : 1 à quatre feuilles, 4 à cinq feuilles, 6 à six feuilles, 15 à sept feuilles, 16 à huit feuilles, 4 à neuf feuilles, 5 à dix feuilles et 3 à onze feuilles. On n'observe donc pas une différence très importante quant au nombre de feuilles par rapport aux échantillons de l'aquarium (tableau II). La principale différence concerne, en effet, l'enracinement : sur les 54 plantules observées à la station, 22 ont atteint un stade " prostré " dans lequel les plantules sont inclinées contre le substrat par la force des racines adventives situées aux nouds des racines qui entourent des morceaux de bois, des galets plus ou moins gros ou des débris variés. Cette abondance de racines adventives sur des substrats tourmentés, hétérogènes, explique en partie l'abondance d'herbiers au niveau de zones de rivières servant plus ou moins de dépotoirs; les possibilités d'accrochage au substrat sont considérablement accrues.

Le développement hétéroblastique des plantules peut être résumé comme suit (fig. 2 a et b) : après les cotylédons, la première feuille présente un limbe découpé en trois lanières capillaires terminales. A la base du pétiole, parfois dès la première feuille, plus fréquemment à partir de la deuxième ou de la troisième, on trouve l'ébauche d'un deuxième limbe identique c'est-à-dire à trois lanières capillaires faiblement pétiolulées. A partir de la cinquième feuille, on observe un troisième limbe pétiolulé à la base du pétiole principal, les deux premiers limbes étant plus grandement développés. Il faut également noter que les bases des premières feuilles n'ont pas de vrai stipules mais plutôt une gaine stipulaire jusqu'à la cinquième ou sixième feuille. L'ensemble de la plantule a un développement hélicoïdal, par ailleurs, à chacun des nœuds se développent en opposition à la feuille une ou plusieurs racines adventives (tableau III) accompagnées de radicelles. La croissance des feuilles peut prendre un développement considérable jusqu'à la feuille adulte, chacun des segments trifides se divisant lui-même en trois parties et ainsi de suite (fig. 2 d), ce qui augmente la biomasse de ces plantes. Par la suite, au printemps, en présence d'un apport nutritif suffisant, un rameau secondaire se développe à l'aisselle des feuilles et assure une ramification érigée qui, à son tour, peut donner d'autres ramifications (fig. $2 \mathrm{c}$ ). La partie prostrée joue alors le rôle d'un rhizome grâce auquel
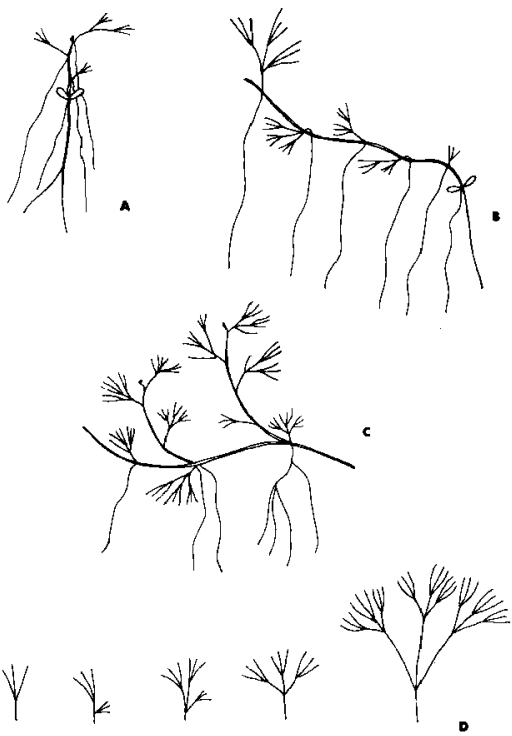

Fig.2. Schéma de la croissance de $R$. penicillatus var. penicillatus.

A. Plantule avec ses cotylédons et des racines adventives à chacun des noeuds cotylédonaire et foliaire.

B. Plantule prostrée amorçant un port érigé.

C. Segments érigés et tige stolonifère.

D. Processus schématique de l'ontogénie foliaire.

se fait la pérennité de l'espèce lorsque l'appareil végétatif est détruit après la floraison. Le rôle de cette partie prostrée ne paraît pas avoir été suffisamment considéré dans le domaine de l'aménagement.

En effet, comme on a pu l'observer maintes fois, les coupes d'herbiers à renoncules (Owens et al. 1961) appor tent parfois des résul tats opposés à ceux escomptés (Mitchell 1974, Westlake \& al. 1982) car elles renforcent la vitalité des rhizomes. Ainsi que l'ont indiqué Westlake (1964), Krause (1977) et Dawson $(1978,1979,1983)$, la réduction de la lumière est plus avantageuse pour limiter l'extension des 
Tábleau II. - Nombre de feuilles observées sur des plantules de Ranunculus penicillatus var. penicillatus âgées de 140 jours, cultivées en aquarium dans des eaux de différentes provenances.

\begin{tabular}{|c|c|c|c|c|c|c|c|c|c|c|c|c|c|c|}
\hline \multirow{2}{*}{$\begin{array}{l}\text { Mélange } \\
\text { eau Lot / } \\
\text { eau Truyère }\end{array}$} & \multicolumn{13}{|c|}{ Nombre de feuilles par plantules } & \multirow{2}{*}{$\begin{array}{c}\text { Total } \\
\text { plantules } \\
\text { observées* }\end{array}$} \\
\hline & 1 & 2 & 3 & 4 & 5 & 6 & 7 & 8 & 9 & 10 & 11 & 12 & 13 & \\
\hline $10 / 0$ & & 4 & 4 & 8 & 17 & 11 & 12 & 8 & 1 & 1 & 1 & & & 67 \\
\hline $8 / 2$ & & & & 3 & 4 & 20 & 10 & 14 & 7 & 7 & 1 & & & 66 \\
\hline $6 / 4$ & 1 & 2 & 9 & 8 & 14 & 4 & 4 & & & & & & & 42 \\
\hline $4 / 6$ & 2 & & 2 & 3 & 7 & 9 & 16 & 6 & 3 & 2 & 1 & & 1 & 52 \\
\hline $2 / 8$ & & & 2 & 4 & 23 & 15 & 8 & 4 & 2 & & & & & 61 \\
\hline $0 / 10$ & & 3 & 4 & 15 & 25 & 14 & 9 & 4 & 1 & & 1 & 1 & & 77 \\
\hline
\end{tabular}

* sur 100 graines semées.

Tableau III. - Nombre de racines adventives observées sur des plantules de Ranunculus penicillatus var. penicillatus âgées de 140 jours cultivées en aquarium dans des eaux de différentes provenances.

\begin{tabular}{crrrrrrr}
\hline $\begin{array}{c}\text { Melange } \\
\text { eau Lot } / \\
\text { eau Truyère }\end{array}$ & \multicolumn{9}{c}{$\begin{array}{c}\text { Nombre de racines adventives } \\
\text { par plantule }\end{array}$} & $\begin{array}{c}\text { Total } \\
\text { plantules } \\
\text { observées }\end{array}$ \\
\hline $10 / 0$ & 1 & 2 & 3 & 4 & 5 & 6 & \\
$8 / 2$ & 7 & 25 & 28 & 7 & & & 67 \\
$6 / 4$ & 3 & 36 & 20 & 6 & 1 & & 66 \\
$4 / 6$ & 7 & 16 & 13 & 3 & 2 & 1 & 42 \\
$2 / 8$ & 2 & 18 & 20 & 8 & 3 & 1 & 52 \\
$0 / 10$ & 2 & 36 & 16 & 2 & 2 & & 61 \\
\hline
\end{tabular}

herbiers, par exemple grâce à l'ombre des arbres du rivage. Pour adapter ce principe aux grandes rivières, nous avons tenté de mettre en place sur le fond des toiles absorbant $50 \%$ de la lumière ambiante. Les résultats obtenus dès la première année étaient encourageants mais l'impossibilité de maintenir ces toiles en place pendant plusieurs saisons (crues) ne permet pas concrètement d'utiliser cette méthode (en aquarium privé de lumière à $50 \%$, l'irréversibilité est atteinte en 28 jours). Au contraire, un arrachage des souches rhizomateuses nous paraîtrait efficace.

Il faut en effet attribuer à ces souches et à leur possibilité de se développer sur des substrats convenables le succès des herbiers à renoncules à l'aval des barrages. Nous avons pu voir ci-dessus que la croissance de $R$. penicillatus var. penicillatus comme celle de $R$. fluitans était variable. Un individu issu d'une semence peut fleurir au bout de trois années en milieu naturel, parfois même d'une année. La floraison peut ètre retardée lorsque les conditions sont mauvaises mais la résistance de la plante est assez surprenante et la faculté de "repartir "vers la floraison peut être conservée très longtemps. Les pêcheurs connaissent bien des populations de renoncules " stabilisées", ne gênant aucunement mais qui, subitement, se développent et émergent à la surface par suite d'une déstabilisation des conditions de milieu : baisse des eaux, apports nut ritifs, ralentissement du courant, nombreux sont les auteurs ayant souligné ces facteurs (Butcher 1933. Holmes et al. 1977 a et $b$, Hynes $1970 \ldots$..). Des phénomènes identiques pourraient se produire dans des lacs de montagne dans lesquels des apports nutritifs actuellement faibles déterminent une stabilité des populations de renoncules.

\section{4. - Conclusion}

Le rôle de la reproduction sexuée est loin d'être négligeable pour les deux espèces étudiées. Il nous 
paraît favorisé par : la grande quantité de semences fertiles, la longévité germinative qui dépasse largement deux ans et finalement la facilité avec laquelle du matériel maintenu à sec peut germer lorsqu'il se retrouve en eau. Les plantules se développent préférentiellement sur des substrats hétérogènes. De nombreuses plantules de $R$. penicillatus var. penicillatus ont ainsi été observées sur des tapis de muscinées : jusqu'à 50 plantules sur une seule touffe de mousse. Une extrêrne plasticité tant en ce qui concerne le développement hétéroblastique que la vitesse de croissance des individus explique l'expansion des deux espèces de Ranunculus en rivière dès que les conditions de substrat sont favorables.

\section{Travaux cités}

Arber (A.). 1920. - Water plonts. A study of aquatic Angiosperms. Cambridge University Press. $436 \mathrm{p}$.

Butcher (R.W.). 1933. - Studies on the ecology of Rivers. 1. On the distribution of macrophytic vegetation in the Rivers of Britain. J. Ecol., $21: 58-91$.

Capblancq (J.) \& Tourenq (J.N.). 1978. - Hydrochimie de la rivière Lot. Annis Limnol., 14 : 25-37.

Cook (C.D.K.). 1966. - A monographic study of Ranunculus subgenus Batrachium (D.C.) A Gray, Mitt. Bot. München, 6 ; 47-237.

Dawson (F.H.). 1978. - Aquatic plant management in semi-natural streams : the role of marginal vegetation. J. Environ. Management, $6: 213-221$

Dawson (F.H.). 1981. - The reduction of light as a technique for the control of aquatic plants, an assessment. Proc. Aquatic Weeds Control: 157.164 .

Dawson (F.H.) \& Haslam (S.M.). 1983. - The management of river vegetation with particular reference to shading effects of marginal vegetation. Landscape Planning, $10: 147-169$.
Dawson (F.H.) \& Kern-Hansen (U.). 1978. - Aquatic weed management in natural streams : the effect of shade by the marginal vegetation. Verh. Intemat. Verein. Limnol, 20 : 1451-1456.

Dawson (F.H.) \& Kern-Hansen (U.). 1979. - The effect of natural and artificial shade on the Macrophytes of lowland streams and the use of shade as a management technique. Int. Revue Ges. Hydrobiol, $64: 437455$.

Decamps (H.), Capblancq (J.), Casanova (H.) \& Tourenq (J.N.). 1979 - Hydrobiology of some regulated rivers in the South West of France. In Ward (J.V.) and Stanford (J.A.) ed. The Ecology of Regulated Streams, Plenurn Press, New.York.

Décamps (O.). 1972. - Monocotylie fréquente chez Ranunculus ihora L. (Renonculacées). C.r. Acad. Sc. Paris, 274 : 838-841.

Décamps (O.). 1978. - Caractères stomatiques des Renonculacées. Bull. Soc. Hist. nat. Toulouse, $114: 429446$.

Décamps (O.). 1979. - Ontogénie nodale chez les Renonculacées. Bull. Soc. bot. Fr., 126:461-471

Holmes (N.T.H.) \& Whitton (B.A.). 1977 a. - The macrophytic vegetation of the river Tees in 1975 : observed and predicted changes. Freshwat. Biol., $7: 43-60$.

Holmes (N.T.H.) \& Whitton (B.A.). 1977 b. - Macrophytes of the river Wear 1966-1976. Naturalist, $102: 53-73$.

Hynes (H.B.N.). 1970 - The Ecology of Rurnting Waters. Liverpool University Press. $555 \mathrm{p}$.

Krause (A.). 1977. - On the effect of marginal tree rows with res. pect to the management of small lowland streams. Aquatic Botany, 3: 185.192

Laville (H.). 1981. - Recoltes d'exuvies nymphales de chironomi. des (Diptera) dans le Haut-Lot, de la source (1 $295 \mathrm{~m}$ ) au confluent de la Truyere (223 m). Annls Limnol., $17: 255-289$.

Mitchell (D.S.) ed. 1974. - Aquatic Vegetation and its use and con. trol. UNESCO, Paris, 135 p.

Owens (M.) \& Edwards (R.N.). 1961. - The effects of plants on river conditions. 2. - Further crop studies and estimates of net productivity of macrophytes in a chalk stream. J. Ecol. 49:119-26.

Westlake (D.F.). 1964. - Light extinction, standing crop and pho tosynthesis within weed beds. Verh. Int. Ver. Limnol., $15: 15.25$

Westlake (D.F.). 1973. - Aquatic macrophyles in rivers. Pol. Arch. Hydrobiol, $20: 31-40$.

Westlake (D.F.) \& Dawson (F.H.). 1982. - Thirty years of weed cutting on a chalk-stream. Proc. EWRS 6ih Symposium on Aquoic Weeds : 132-140.

Whit ton (B.A.) \& Buckmaster (R.C.). 1970. Macrophytes of the River Wear. Naturalist Bull., $914: 97.116$. 